\title{
A Simulation Study on the Effects of Media Composition on the Growth Rate of Escherichia coli MG1655 Using iAF1260 Model
}

\section{Kar Chi Cheong ${ }^{1}$, Raphael YH Hon ${ }^{1}$, Clara J Sander ${ }^{1}$, Irwin ZL Ang ${ }^{1}$, Jun Hang Foong ${ }^{1}$ and Maurice HT Ling ${ }^{1,2 *}$}

${ }^{1}$ School of Applied Sciences, Temasek Polytechnic, Singapore

${ }^{2}$ HOHY PTE LTD, Singapore

*Corresponding Author: Maurice HT Ling, School of Applied Sciences, Temasek

Polytechnic and HOHY PTE LTD, Singapore.
Received: June 22, 2020

Published: July 22, 2020

(C) All rights are reserved by Maurice HT Ling., et al.

\begin{abstract}
Media compositions are important determinants of growth rate and genome-scale models (GSMs) had been used for optimizing media for metabolite production and growth. Recently, iAF1260, a GSM based on Escherichia coli MG1655, was used to study the effects varying glucose concentration in media on growth rate and metabolic fluxes. In this study, the effects of other media components in the presence of varying glucose concentrations on the predicted growth rate of E. coli MG1655 were examined. Our results show that 10 media components (ammonium, calcium, chloride, copper, glucose, manganese, magnesium, molybdate, phosphate, and potassium) demonstrate substantial impact on the predicted growth rate of E. coli MG1655. Of which, 4 components (glucose, ammonium, magnesium, and phosphate) have the most impact. However, our results also demonstrate the limitations of iAF1260 as media components that had been shown to affect E. coli growth rate were not reflected by the model.
\end{abstract}

Keywords: Growth Rate; Genome-Scale Models; Media Optimization

\section{Introduction}

Growth rate of cells in different media [1] is an important physiological parameter as it can affect various experiments [2]; such as, antibiotics [3,4] and antibacterial [5] susceptibilities. A recent study has also shown that the types of growth media can affect the metabolomics of Escherichia coli [6]. This is supported by studies showing that media compositions can affect the production of enzymes [7]. This led to studies on media optimization for various purposes: such as, production of uricase from Bacillus subtilis [8], production of succinic acid by Enterobacter sp. LU1 [9], and production of isocitric acid by Yarrowia lipolytica [10].

Genome-scale models (GSMs) had been used in various applications [11]; such as, metabolic engineering for specific metabolite production [12-14]. Since bacterial growth rate can be affected by media composition $[1,15]$, it is plausible to estimate the effects of media compositions on growth rate [16] as that demonstrated by Chen., et al [17].

Previously, the GSM iAF1260 [18] had been used to show correlation of $E$. coli growth rate and metabolic fluxes on various glucose concentrations [19] as E. coli is an experimental organism used in various applications [20]. In this study, we examine the effects of other media components in the presence of varying glucose concentrations on predicted growth rate of E. coli MG1655. Our results suggest that 4 media components (glucose, ammonium, magnesium, and phosphate) have the most impact on the predicted growth rate of E. coli. However, our results also demonstrate limitations of GSM iAF1260 [18] as media components that had been shown to affect $E$. coli growth rate were not reflected by the model. 


\section{Materials and Methods}

GSM Model: Simulated metabolism data was obtained by Parsimonious Flux Balance Analysis (pFBA) [23] using Cameo [21] via AdvanceSyn Toolkit (https://github.com/mauriceling/advancesyntoolkit) on iAF1260 model [18], a GSM based on E. coli MG1655 [18], from the BiGG database [22].

Sensitivity analysis of media components: Sensitivity analysis on 19 media components [(i) calcium (EX_ca2_e), (ii) chloride (EX_ cl_e), (iii) carbon dioxide (EX_co2_e), (iv) cobalt (EX_cobalt2_e), (v) copper (EX_cu2_e), (vi) ferrous (EX_fe2_e), (vii) ferric (EX_ fe3_e), (viii) water (EX_h2o_e), (vix) proton (EX_h_e), (x) potassium (EX_k_e), (xi) magnesium (EX_mg2_e), (xii) manganese (EX_ mn2_e), (xiii) molybdate (EX_mobd_e), (xiv) sodium (EX_na1_e), (xv) ammonium (EX_nh4_e), (xvi) phosphate (EX_pi_e), (xvii) sulfate (EX_so4_e), (xvii) tungstate (EX_tungs_e), and (xiv) zinc (EX_zn2_e)] defined was performed to investigate the relative effects of each component on the growth rate of the cell as determined by the corresponding objective value from the model using pFBA [23] Cameo [21]. The sensitivity of each media component is $\sqrt{\sum_{i=0}^{N}\left(x_{i}-599.768\right)^{2}}$, where is the objective value after changing one media influx rate at a time $(24,25)$ and 599.768 is the objective value using the native media composition. The intake fluxes used for the different compounds were $0,0.01,0.1,1,10,100$, and 1000 mmol per gram dry weight per hour (mmol/gDW/hr). Hence, the sensitivity score is directly proportional to the impact of the media component to the growth rate of the cell.

Effects of multiple media components on growth rate: The effects of media components on growth rate was determined using stepwise regression using Akaike information criterion [26] from MASS [27] as Objective value $=\sum_{i=1}^{N} \beta_{i}$ Media $_{i}+\beta_{0}$, where $\beta_{i}$ is the coefficient of $\mathrm{i}$-th media component, and $\beta_{o}$ is the constant value. A total of 200 combinations of concentrations with regular intervals of $0.05 \mathrm{mmol} / \mathrm{gDW} / \mathrm{hr}$ ranging from 0.025 to $9.975 \mathrm{mmol} / \mathrm{gDW} /$ hr were determined using Latin Hypercube Design [28] in pyDOE (https://pythonhosted.org/pyDOE/).

\section{Results and Discussion}

Our results show that glucose cannot be absent as it results in an error in pFBA [23], suggesting a mandatory requirement for carbon source. Of the 19 non-carbon media components analyzed for impact on $E$. coli growth rate using one-factor-a-time sensitivity

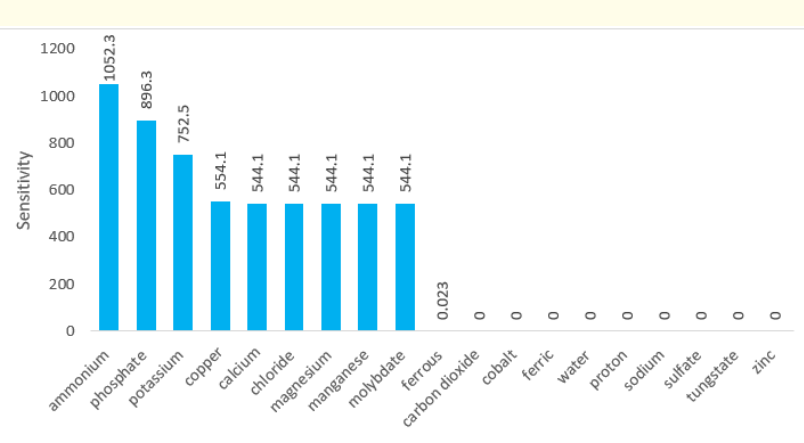

Figure 1: Effects of 19 non-carbon media components on growth rate.

analysis [24,25] suggests that only nine components exhibit substantial impact on growth rate (Figure 1). These nine components are (i) ammonium, (ii) calcium, (iii) chloride, (iv) copper, (v) manganese, (vi) magnesium, (vii) molybdate, (viii) phosphate, and (ix) potassium. These components largely corresponds to (Figure 2) the standard M9 minimal media [29], which contains (i) ammonium, (ii) calcium, (iii) chloride, (iv) magnesium, (v) phosphate, (vi) potassium, (vii) sulfate, (viii) sodium, and (vix) glucose.

Of the 200 combinations of media components determined using Latin Hypercube Design [28], 193 combinations gave non-error objective values, which were then used in regression analysis. Regression analysis on the 10 media components, including glucose, with impact on $E$. coli growth rate (Table 1; full model) suggests significant correlation (adjusted $r^{2}=0.6052, F=30.43, p$-value $<$ $2.2 \mathrm{e}-16)$ between these 10 media components and the predicted growth rate. However, only two coefficients (glucose and ammonium) are significant ( $p$-value $<2 \mathrm{e}-16)$. Stepwise regression using Akaike information criterion [26] from MASS [27] suggests four media components; namely, glucose, ammonium, magnesium, and phosphate; with the largest impact to E. coli growth rate (Table 2; reduced model). The correlation between the reduced model is slightly higher than that of the full model (adjusted $\mathrm{r}^{2}=0.6137, \mathrm{~F}$ $=77.27$, $\mathrm{p}$-value $<2.2 \mathrm{e}-16$ ). This is consistent with a review by Peterson., et al. [30] suggests that starvation of one of the three nutrients; namely, carbon (in the form of glucose), nitrogen (in the form of ammonium), or phosphorus (in the form of phosphate); triggers E. coli into dormancy. Thus, suggesting the importance of glucose, ammonium, and phosphate to $E$. coli growth. The dependence of $E$. 


\begin{tabular}{|c|c|c|c|c|}
\hline Regressor & Coefficient & Standard Error & t-statistic & p-value \\
\hline (Intercept) & -107.174 & 43.3698 & -2.471 & 0.0144 \\
\hline Glucose & 27.749 & 2.7887 & 9.951 & $<2 \mathrm{e}-16$ \\
\hline Manganese & 0.8339 & 2.723 & 0.306 & 0.7598 \\
\hline Copper & 1.0141 & 2.7096 & 0.374 & 0.7087 \\
\hline Potassium & 0.9016 & 2.7018 & 0.334 & 0.7390 \\
\hline Magnesium & 5.4352 & 2.6568 & 2.046 & 0.0422 \\
\hline Calcium & -2.985 & 2.6552 & -1.124 & 0.2624 \\
\hline Chloride & 1.0118 & 2.6852 & 0.377 & 0.7067 \\
\hline Molybdate & 0.3652 & 2.724 & 0.134 & 0.8935 \\
\hline Ammonium & 40.7948 & 2.6902 & 15.164 & $<2 \mathrm{e}-16$ \\
\hline Phosphate & 3.7352 & 2.6984 & 1.384 & 0.1680 \\
\hline
\end{tabular}

Table 1: Impact of media components on growth rate (Full Model).

\begin{tabular}{|c|c|c|c|c|}
\hline Regressor & Coefficient & Standard Error & t-statistic & p-value \\
\hline (Intercept) & -100.522 & 27.754 & -3.622 & 0.0004 \\
\hline Glucose & 27.282 & 2.708 & 10.075 & $<2 \mathrm{e}-16$ \\
\hline Magnesium & 5.641 & 2.608 & 2.163 & 0.0328 \\
\hline Ammonium & 40.757 & 2.63 & 15.497 & $<2 \mathrm{e}-16$ \\
\hline Phosphate & 3.858 & 2.609 & 1.479 & 0.1409 \\
\hline
\end{tabular}

Table 2: Impact of media components on growth rate (Reduced Model).

coli growth on extracellular magnesium is illustrated in the 1960s by McCarthy [31] and Lusk., et al. [32] demonstrating the importance of magnesium in maintaining ribosome function.

Given that M9 minimal media can be considered as the minimum set of nutrients required for $E$. coli growth, it is conceivable to expect that all components of M9 minimal media should impact on E. coli growth rate. That is, M9 minimum media components should be a subset of growth sensitive components. Hence, the presence of M9 minimal media components (sodium and sulfate) not identified by iAF1260 [18] to be impacting on growth rate (Figure 2) may indicate limitations of the model.

Importantly, our results show an objective value rather than an error in pFBA [23] when sulfate intake is set to zero to indicate the void of sulfate in the media, which suggests that sulfate is a non-requirement. This contradicts Kertesz and Cook [33], whom suggest an absolute requirement for sulfate and found a set of proteins induced as a result of sulfate limitation in E. coli. Similarly, sodium chloride has been shown to affect the growth rate of $E$. coli

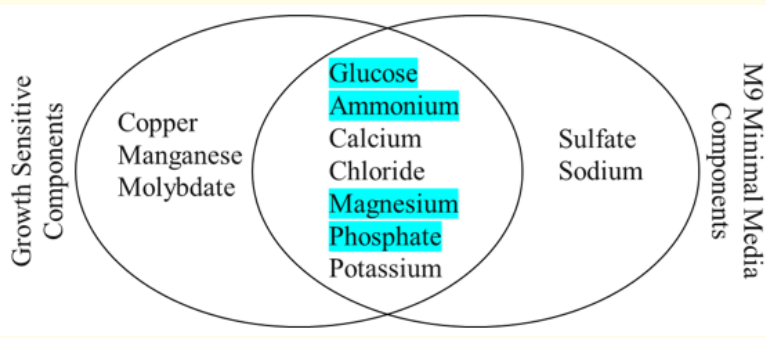

Figure 2: Commonalities between growth sensitive components and $\mathrm{m} 9$ minimal media [29] components. The 4 media components having the most impact on the predicted growth rate of $\mathrm{E}$. coli are highlighted.

[34-36]. Although it may be possible to argue that effects of sodium chloride on growth rate can be confounded by chloride, which is a growth sensitive component; a study by Dupree., et al. [37] suggest differential effects of sodium chloride and calcium chloride on the growth of $E$. coli. This suggests that the effects of calcium, 
sodium, and chloride on E. coli growth rate is likely to be independent; hence, the effects of sodium being confounded by the effects of chloride is not likely.

\section{Conclusion}

Therefore, this study highlights a fundamental and crucial divergence between experimental results and simulation results. This is supported by a study by Khodayari and Maranas [38] suggesting poor correlation between experimental yields and simulated yields using iAF1260 [18]. This may have implications on the applications of iAF1260 [18] for media optimizations. However, this study also provides a direction to improve on iAF1260 [18] by focus on improving the correlation and minimizing the error between experimental and predicted growth rates in different media.

\section{Author's Contribution}

KCC, RYH, CJS, IZA, and JHF contributed equally to this study.

\section{Conflict of Interest}

The authors declare no conflict of interest.

\section{Bibliography}

1. Miyashira $\mathrm{CH}$. , et al. "Comparison of radial growth rate of the mutualistic fungus of Atta sexdens rubropilosa forel in two culture media". Brazilian Journal of Microbiology 41.2 (2010): 506-511.

2. Bich GA., et al. "Isolation of the symbiotic fungus of Acromyrmex pubescens and phylogeny of Leucoagaricus gongylophorus from leaf-cutting ants". Saudi Journal of Biological Science 24.4 (2017): 851-856.

3. Haugan MS., et al. "Comparative Activity of Ceftriaxone, Ciprofloxacin, and Gentamicin as a Function of Bacterial Growth Rate Probed by Escherichia coli Chromosome Replication in the Mouse Peritonitis Model". Antimicrobe Agents Chemotherapy 63.2 (2019): e02133-02118.

4. Greulich P., et al. "Growth-dependent bacterial susceptibility to ribosome-targeting antibiotics". Molecular Systems Biology 11.3 (2015): 796.

5. De Leersnyder I., et al. "Influence of growth media components on the antibacterial effect of silver ions on Bacillus subtilis in a liquid growth medium". Science Report 8.1 (2018): 9325.
6. Ratiu I-A., et al. "The effect of growth medium on an Escherichia coli pathway mirrored into GC/MS profiles". Journal of Breath Research 11.3 (2017): 036012.

7. Tavafi H., et al. "Screening of alginate lyase-producing bacteria and optimization of media compositions for extracellular alginate lyase production". Iran Biomed Journal 21.1 (2017): 48-56.

8. Pustake SO., et al. "Statistical media optimization for the production of clinical uricase from Bacillus subtilis strain SP6". Heliyon 5.5 (2019): e01756.

9. Podleśny M., et al. "Media optimization for economic succinic acid production by Enterobacter sp. LU1". AMB Express 7.1 (2017): 126.

10. Kamzolova SV., et al. "Fermentation Cconditions and media optimization for isocitric acid Production from ethanol by Yarrowia lipolytica". BioMed Research International (2018): 2543210.

11. McCloskey D., et al. "Basic and applied uses of genome-scale metabolic network reconstructions of Escherichia coli". Molecular Systems Biology 9 (2013): 661.

12. Mishra P., et al. "Genome-scale model-driven strain design for dicarboxylic acid production in Yarrowia lipolytica". BMC System Biology 12 (2018): 12.

13. Iranmanesh E., et al. "Improving l-phenylacetylcarbinol production in Saccharomyces cerevisiae by in silico aided metabolic engineering". Journal of Biotechnology 308 (2020): 27-34.

14. Kim M., et al. "In silico identification of metabolic engineering strategies for improved lipid production in Yarrowia lipolytica by genome-scale metabolic modelling". Biotechnology and Biofuels 12 (2019): 187.

15. Ashino K., et al. "Predicting the decision making chemicals used for bacterial growth". Science Report 9.1 (2019): 7251.

16. O’Brien EJ., et al. "Using genome-scale models to predict biological capabilities". Cell 161.5 (2015): 971-987.

17. Chen Y., et al. "An unconventional uptake rate objective function approach enhances applicability of genome-scale models for mammalian cells". npj Systems Biology and Applications 5 (2019): 25. 
18. Feist AM., et al. "A genome-scale metabolic reconstruction for Escherichia coli K-12 MG1655 that accounts for 1260 ORFs and thermodynamic information". Molecular Systems Biology 3 (2007): 121.

19. Chang ED and Ling MH. "Explaining Monod in terms of Escherichia coli metabolism". Acta Scientific Microbiology 2.9 (2019): 66-71.

20. Cronan JE. "Escherichia coli as an experimental organism". In: John Wiley and Sons Ltd, editor. eLS [Internet]. Chichester, UK: John Wiley and Sons, Ltd (2014).

21. Cardoso JGR., et al. "Cameo: A Python library for computer aided metabolic engineering and optimization of cell factories". ACS Synthetic Biology 7.4 (2018): 1163-1166.

22. King ZA., et al. "BiGG Models: A platform for integrating, standardizing and sharing genome-scale models". Nucleic Acids Research 44 (2016): D515-522.

23. Lewis NE., et al. "Omic data from evolved E. coli are consistent with computed optimal growth from genome-scale models". Molecular Systems Biology 6 (2010): 390.

24. Abou-Taleb KA and Galal GF. "A comparative study between one-factor-at-a-time and minimum runs resolution-IV methods for enhancing the production of polysaccharide by Stenotrophomonas daejeonensis and Pseudomonas geniculate". Annuals of Agriculture Science 63.2 (2018): 173-180.

25. Razavi S and Gupta HV. "What do we mean by sensitivity analysis? The need for comprehensive characterization of "global" sensitivity in Earth and Environmental systems models: A Critical Look at Sensitivity Analysis". Water Resource Research 51.5 (2015): 3070-3092.

26. Akaike H. "Information theory and an extension of the maximum likelihood principle". In: Selected Papers of Hirotugu Akaike. Springer (1998): 199-213.

27. Ripley B., et al. "Package 'mass'”. Cran R (2013)

28. Viana FAC. "A tutorial on Latin Hypercube Design of experiments". Quality and Reliability Engineering International 32.5 (2016): 1975-85.

29. M9 minimal medium (standard). Cold Spring Harbor Protocol (2010): pdb.rec12295.
30. Peterson CN., et al. "Escherichia coli starvation diets: Essential nutrients weigh in distinctly". Journal of Bacteriology 187.22 (2005): 7549-7553.

31. McCarthy BJ. "The effects of magnesium starvation on the ribosome content of Escherichia coli". 55.6 (1962): 880-889.

32. Lusk JE., et al. "Magnesium and the growth of Escherichia coli". Journal of Biological Chemistry 243.10 (1968): 2618-2624.

33. Kertesz MA., et al. "Proteins induced by sulfate limitation in Escherichia coli, Pseudomonas putida, or Staphylococcus aureus". Journal of Bacteriology 175.4 (1993): 1187-1190.

34. Omotoyinbo $\mathrm{O}$ and Omotoyinbo B. "Effect of varying $\mathrm{NaCl}$ concentrations on the growth curve of Escherichia coli and Staphylococcus aureus". Cell Biology 4.5 (2016): 31-34.

35. Goh DJ., et al. "Gradual and step-wise halophilization enables Escherichia coli ATCC 8739 to adapt to $11 \% \mathrm{NaCl}$ ". Electron Physician 4.3 (2012): 527-535.

36. How JA., et al. "Adaptation of Escherichia coli ATCC 8739 to 11\% NaCl". Dataset Papers in Biology (2013).

37. Dupree DE., et al. "Effects of sodium chloride or calcium chloride concentration on the growth and survival of Escherichia coli 0157:H7 in model vegetable fermentations". Journal of Food Protection 82.4 (2019): 570-578.

38. Khodayari A and Maranas CD. "A genome-scale Escherichia coli kinetic metabolic model k-ecoli457 satisfying flux data for multiple mutant strains". Nature Communication 7 (2016): 13806.

\section{Assets from publication with us}

- Prompt Acknowledgement after receiving the article

- Thorough Double blinded peer review

- Rapid Publication

- Issue of Publication Certificate

- High visibility of your Published work

Website: www.actascientific.com/

Submit Article: www.actascientific.com/submission.php Email us: editor@actascientific.com

Contact us: +919182824667 